\title{
ON THE SIZE OF THE SET OF LEFT INVARIANT MEANS ON A SEMIGROUP
}

\author{
CHING $\mathrm{CHOU}^{1}$
}

1. Introduction. Let $S$ be a semigroup with discrete topology, $m(S)$ the space of bounded real functions on $S$ with the sup. norm, and $m(S)^{*}$ the conjugate Banach space of $m(S) . \phi^{\prime} \in m(S)^{*}$ is a mean if $\left\|\phi^{\prime}\right\|=1$, and $\left(\phi^{\prime}, f\right) \geqq 0$ whenever $f \geqq 0$. A mean $\phi^{\prime}$ is said to be left invariant if $\left(\phi^{\prime}, l_{s} f\right)=\left(\phi^{\prime}, f\right)$ for all $s \in S$ and $f \in m(S)$, where the left translation $l_{s}$ of $m(S)$ by $s$ is given by $\left(l_{s} f\right)\left(s_{1}\right)=f\left(s s_{1}\right)$. The set of left invariant means on $S$ is denoted by $M l^{\prime}(S)$. If $M l^{\prime}(S)$ is not empty, we say $S$ is left amenable.

When $S$ is left amenable it is natural to ask how big is the set $M l^{\prime}(S)$. Day [1] started the investigation by showing that an infinite abelian group has more than one left invariant means. Luthar [6] and Granirer [4], [5] then searched for the necessary and sufficient conditions for a semigroup to have a unique left invariant mean. Among other things, they obtained the following two results:

(1) If $S$ is any abelian semigroup with no finite ideal (which is the case if $S$ has cancellation and is infinite) then there are $\phi^{\prime}, \psi^{\prime} \in M l^{\prime}(S)$ such that $\left\|\phi^{\prime}-\psi^{\prime}\right\|=2$, cf. [6];

(2) If $S$ is an infinite left amenable left cancellation semigroup, then the dimension of the linear span of $M l^{\prime}(S)$ is infinite, cf. [4]. The purpose of this paper is to prove the following

THEOREM. Let $S$ be an infinite left amenable semigroup with cancellation. Then there exists a set $E \subset M l^{\prime}(S)$ satisfying:

(1) card $E=2^{c}$ card $S$, where $c$ is the cardinality of the continuum,

(2) each $\phi^{\prime} \in E$ is an extreme point of $M l^{\prime}(S)$, and

(3) if $\phi^{\prime}, \psi^{\prime} \in E, \phi^{\prime} \neq \psi^{\prime}$, then $\left\|\phi^{\prime}-\psi^{\prime}\right\|=2$.

This theorem is a generalization of the results quoted above for the case that $S$ has cancellation.

For (3) we shall actually construct the set $E$ in such a way that for distinct $\phi^{\prime}$ and $\psi^{\prime}$ in $E$, their supports are disjoint, considered as measures on the Stone-Čech compactification of the discrete set $S$.

Since every left amenable, right cancellation, finite semigroup is

Received by the editors January 17, 1969.

${ }^{1}$ Part of the results in this paper is contained in the Ph.D. thesis of the author under the direction of R. A. Raimi at the University of Rochester. The author wishes to thank Professor Raimi for his encouragement and advice. 
actually a group and every finite group has a unique left invariant mean, we have as a consequence of the above theorem the following

Corollary. Let $S$ be a left amenable semigroup with cancellation. Then card $M l^{\prime}(S)=1$ or $\geqq 2^{c}$. It is one if and only if $S$ is finite (and hence is a group).

By our theorem, the additive semigroups of positive integers has exactly $2^{c}$ left invariant means. Even for this special case, our result seems to be new.

2. Preliminaries and notation. In this paper we only consider semigroups with discrete topology. Let $S$ be a semigroup. The StoneCech compactification of $S$ is denoted by $\beta S$. It is well known that $m(S)$ and $C(\beta S)$, the space of real-valued continuous functions on $\beta S$, are isomorphic as Banach spaces. If $\phi^{\prime} \in m(S)^{*}$, then it corresponds to a unique real Borel measure $\phi$ on $\beta S$. The correspondence is characterized by $\left(\phi^{\prime}, f\right)=\int_{\beta S} \bar{f} d \phi$, where $f \in m(S)$ and $\bar{f}$ denotes its continuous extension to $\beta S$. In the sequel, for $\phi^{\prime} \in m(S)^{*}, \phi$ will always denote the corresponding measure. In particular, we denote by $M l(S)$ the set of measures corresponding to $M l^{\prime}(S)$.

In this paragraph we shall collect some topological facts of the space $\beta S$. These facts will be used later without any further explanation. If $B \subset S, B^{-}$will denote the closure of $B$ in $\beta S$. Sets of the form $B^{-}, B \subset S$, are closed-open in $\beta S$ and they form a topological open basis for $\beta S$. For $B C S, B^{\wedge}$ will denote the set $B^{-} \backslash S$. In particular, $S^{\wedge}=\beta S \backslash S . S^{\wedge}$ is a closed subset of $\beta S$ and sets of the form $B^{\wedge}, B \subset S$, are closed-open in $S^{\wedge}$, and they form a topological open basis for $S^{\wedge}$. For two subsets $A, B \subset S, A^{\wedge} \cap B^{\wedge}=\varnothing$ if and only if $A \cap B$ is finite and $A^{\wedge}=B^{\wedge}$ if and only if $(A \backslash B) \cup(B \backslash A)$ is finite. All of the above facts can be proved easily and can be found in Gillman and Jerison [3].

For each $s \in S$, we have a continuous mapping $s^{\sim}$ of $S$ into $\beta S$ defined by $s \sim\left(s_{1}\right)=s s_{1}, s_{1} \in S$. $s^{\sim}$ has a unique continuous extension to $\beta S$. The extended mapping will also be denoted by $s^{\sim}$. We shall need the following

Lemma 2.1. Let $S$ be an infinite left cancellation semigroup. Then:

(1) For $s \in S$ and $B C S, s^{\sim} B^{\wedge}=(s B)^{\wedge}$. In particular, $s^{\sim} S^{\wedge} \subset S^{\wedge}$.

(2) For each $s \in S, s^{\sim}$ is one-one on $\beta S$.

Proof. (1) Let $\omega \in B^{\wedge}$. Then there is a net $s_{\alpha}$ in $B$ such that $\omega=\lim s_{\alpha}$. Since $s^{\sim}$ is continuous, $s^{\sim} \omega=\lim s^{\sim} s_{\alpha} \in(s B)^{-}$. On the other hand, since $\omega \notin S, s_{\alpha}$ is not eventually a constant. But $S$ satisfies the 
left cancellation law, the net $s \sim s_{\alpha}=s s_{\alpha}$ is not eventually a constant either. Thus $s^{\sim} \omega=\lim s^{\sim} s_{\alpha} \notin S$, since $S$ is discrete, and hence, $s^{\sim} \omega$ $\in(s B)^{\wedge}$.

Conversely, assume $\omega_{1}=\lim s s_{\alpha} \in(s B)^{\wedge}$, where $s_{\alpha} \in B$ for each $\alpha$. Choose a subnet $s_{\beta}$ such that $\omega=\lim s_{\beta}$ exists. Clearly $\omega \in S$. Thus $\omega_{1}=\lim s s_{\beta}=s^{\sim} \omega \in s^{\sim} B^{\wedge}$.

(2) Assume $\omega_{1}, \omega_{2} \in \beta S, \omega_{1} \neq \omega_{2}$. Choose a subset $B \subset S$ such that $\omega_{1} \in B^{-}$and $\omega_{2} \in(S \backslash B)^{-}$. Then $s^{\sim} \omega_{1} \in(s B)^{-}$and $s^{\sim} \omega_{2} \in(s(S \backslash B))^{-}$. Now since $S$ has left cancellation, $s B \cap s(S \backslash B)=\varnothing$ and hence, $(s B)^{-} \cap(s(S \backslash B))^{-}=\varnothing$. Thus $s^{\sim} \omega_{1} \neq s^{\sim} \omega_{2}$ and $s^{\sim}$ is one-one on $\beta S$.

Notation. If $X$ is a convex subset of a vector space, then ex $X$ denotes the set of all extreme points of $X$.

If $\mu$ is a Borel measure on a compact space then the support of $\mu$ will be denoted by supp $\mu$.

If $A$ is a set, $|A|$ will denote the cardinality of $A$.

\section{The main theorem.}

MAIN THEOREM. Let $S$ be an infinite left amenable semigroup with cancellation. Then there is a set $E \subset M l(S)$ such that

(1) $|E| \geqq 2^{c}|S|$,

(2) $E \subset \operatorname{ex} M l(S)$, and

(3) $\phi, \psi \in E, \phi \neq \psi$, implies supp $\phi \cap \operatorname{supp} \psi=\varnothing$.

Definition 3.1. Let $S$ be a semigroup. $A$ set $X \subset \beta S$ is said to be invariant if $s \sim X \subset X$ for each $s \in S$.

Definition 3.2. Let $S$ be a semigroup. A set $A \subset S$ is said to be thin if $s_{1} A \cap s_{2} A$ is finite for each pair of distinct elements $s_{1}, s_{2}$ in $S$.

It is easy to see that the main theorem is a consequence of the following three lemmas:

LEMma 1. If $S$ is an infinite left amenable semigroup with cancellation then there exists a thin set $A \subset S$ with $|A|=|S|$.

LEMma 2. If a cancellation semigroup $S$ contains an infinite thin set $A$ then there are at least $2^{c} \cdot|A|$ nonempty, mutually disjoint, closed, invariant subsets of $\beta S$.

Lemma 3. Let $S$ be a left amenable semigroup. If $K$ is a nonempty, closed, invariant subset of $\beta S$ then there exists a point $\phi \in \operatorname{ex} M l(S)$ such that supp $\phi \subset K$.

4. Proof of Lemma 1. It is well known that if $S$ is a left amenable semigroup with cancellation then $S$ can be embedded into a group, 
cf. [7, Corollary 3.6]. Thus Lemma 1 is a consequence of the following

Proposition 4.1. Assume that $S$ is an infinite subsemigroup of a group $G$. Then $S$ contains a thin set $A$ with $|A|=|S|$.

Proof. We shall construct such a set $A$ by transfinite induction. If $\alpha$ is an ordinal number, then $|\alpha|$ will denote the cardinality of $\alpha$. Let $\alpha_{0}$ be the first ordinal number satisfying $\left|\alpha_{0}\right|=|S|$.

Choose $x_{1} \in S$. Assume $\alpha$ is an ordinal number, $\alpha<\alpha_{0}$, and assume that we have chosen $x_{\beta} \in S$ for all $\beta<\alpha$. Set

$$
X_{\alpha}=\left\{y_{\beta_{1}} y_{\beta_{2}} y_{\beta_{3}}: \beta_{i}<\alpha \text { and } y_{\beta_{i}}=x_{\beta_{i}} \text { or } x_{\beta_{i}}^{-1}\right\} \text {. }
$$

Note that if $\alpha$ is finite then $X_{\alpha}$ is finite and if $\alpha$ is not finite then $\left|X_{\alpha}\right|=|\alpha|<\left|\alpha_{0}\right|$. Thus we can choose $x_{\alpha} \in S \backslash X_{\alpha}$. Thus by induction, we have a set $A=\left\{x_{\alpha}: \alpha<\alpha_{0}\right\} \subset S$ with $|A|=|S|$.

We want to show that $A$ is a thin set in $S$. Indeed, fix $s_{1}, s_{2} \in S, s_{1} \neq s_{2}$, and then fix an element $s_{1} x_{\alpha}=s_{2} x_{\beta} \in s_{1} A \cap s_{2} A$, if there is any. Now let $s_{1} x_{\alpha_{1}}=s_{2} x_{\beta_{1}}$ be an arbitrary element in $s_{1} A \cap s_{2} A$. Then $s_{2}^{-1} s_{1}=x_{\beta} x_{\alpha}^{-1}$ $=x_{\beta_{1}} x_{\alpha_{1}}^{-1}$. If there is an ordinal number $\gamma \in \Gamma=\left\{\alpha, \beta, \alpha_{1}, \beta_{1}\right\}$ such that $\gamma$ is strictly larger than all the other elements of $\Gamma$, then $x_{\gamma} \in X_{\gamma}$ which is a contradiction. Since $s_{1} \neq s_{2}$ we have $\alpha \neq \beta$ and $\alpha_{1} \neq \beta_{1}$. Thus there are only three possible relations between the pairs $(\alpha, \beta),\left(\alpha_{1}, \beta_{1}\right):(1)$ $\alpha_{1}=\alpha$ and $\beta_{1}=\beta$, (2) $\beta_{1}=\alpha$ and then $\alpha_{1}$ is uniquely determined by $x_{\alpha_{1}}=x_{\alpha} x_{\beta}^{-1} x_{\alpha}$, and (3) $\alpha_{1}=\beta$; then $\beta_{1}$ is uniquely determined by $x_{\beta_{1}}=x_{\beta} x_{\alpha}^{-1} x_{\beta}$. Thus we have actually proved that if $s_{1} \neq s_{2}$ then $\left|s_{1} A \cap s_{2} A\right| \leqq 3$.

\section{Proof of Lemma 2.}

Notations. Let $S$ be a semigroup. If $\omega \in S^{\wedge}$, the set $o(\omega)=\{s \sim \omega$ : $s \in S\}$ is called the orbit of $\omega$. Note that both $o(\omega)$ and $o(\omega)^{-}$, the closure of $o(\omega)$, are invariant and if $S$ is of left cancellation then $o(\omega)-C S^{\wedge}$ (Lemma 2.1).

Let $A$ be an infinite thin set in a cancellation semigroup $S$. Then by the definition we know $\left(s_{1} A\right)^{\wedge} \cap\left(s_{2} A\right)^{\wedge}=\varnothing$ if $s_{1} \neq s_{2}$. But by Lemma 2.1, this means that $s_{1} \sim A^{\wedge} \cap s_{2} \sim A^{\wedge}=\varnothing$. Thus for each $\omega \in A^{\wedge}$, the set $o(\omega)$ is discrete in subspace topology. Moreover, if $\omega_{1}, \omega_{2} \in A^{\wedge}$ and $\omega_{1} \neq \omega_{2}$ then we can find $A_{1}, A_{2} \subset A$ such that $\omega_{i} \in \hat{A_{i}}, i=1,2$, and $\hat{A_{1}} \cap \hat{A_{2}}=\varnothing$. Note that for each $s \in S, \hat{s} \hat{A_{1}} \cap \hat{s^{\sim}} \hat{A_{2}}=\varnothing$ and $s \sim \omega_{i} \in s^{\sim} \hat{i_{i}}, i=1,2$. Thus $o\left(\omega_{1}\right) \cap o\left(\omega_{2}\right)=\varnothing$. It is even natural to believe that $o\left(\omega_{1}\right)^{-} \cap o\left(\omega_{2}\right)^{-}=\varnothing$.

Conjecture. Let $A$ be an infinite thin subset of a cancellation semigroup $S$. Then $\omega_{1}, \omega_{2} \in A^{\wedge}, \omega_{1} \neq \omega_{2}$ implies $o\left(\omega_{1}\right)^{-} \cap o\left(\omega_{2}\right)^{-}=\varnothing$. 
If this conjecture would be true then since $A^{\wedge}$ contains $2^{2^{|A|}}$ points $\left(A^{\wedge}=A^{-} \backslash A\right.$, and $A^{-}$is homeormorphic to $\beta A$ which contains $2^{2^{|A|}}$ points, cf. $[3$, p. 130]) we would have the following improved version of Lemma 2:

If $A$ is an infinite thin subset of a cancellation semigroup $S$ then $S$ has at least $2^{2^{|\boldsymbol{\Lambda}|}}$ nonempty, mutually, disjoint closed, invariant sets.

And consequently, we would have a better main theorem, namely, we could change (1) in the main theorem to:

$(1)^{\prime}|E|=2^{2^{|S|}}$.

Unfortunately, we can only prove the conjecture for the case $|A|=\boldsymbol{N}_{\text {o. }}$.

Proposition 5.1. Let $A$ be an infinite thin set of a cancellation semigroup $S$. Set $C=\left\{\omega \in A^{\wedge}: \omega \in D^{\wedge}\right.$ for a certain countable subset $\left.D \subset S\right\}$. Then for $\omega_{1}, \omega_{2} \in C, \omega_{1} \neq \omega_{2}$, we have o $\left(\omega_{1}\right)^{-} \cap o\left(\omega_{2}\right)^{-}=\varnothing$.

The set $A$ can be divided into $|A|$ mutually disjoint sets $A_{\alpha}$ where for each $\alpha,\left|A_{\alpha}\right|=\boldsymbol{\aleph}_{0}$. Now $A_{\alpha}^{\hat{~}}$ contains $2^{c}$ points (cf. [3, p. 131]) and hence $U \hat{A_{\alpha}}$ contains $2^{c} \cdot|A|$ points. But $\cup \hat{A_{\alpha}} \subset C$, and, therefore, Lemma 2 is a consequence of Proposition 5.1.

Proof of Proposition 5.1. Let $B$ be a countably infinite subset of $A$. We define an equivalence relation on $S$ as follows: $s \sim t$ if and only if there exist finitely many elements $s_{1}, \cdots, s_{n}$ in $S$ such that $s_{1}=s, s_{n}=t$, and $s_{i} B \cap s_{i+1} B \neq \varnothing$ for $i=1,2, \cdots, n-1$. For each fixed $s \in S$, there exist at most countably many $t \in S$ such that $s B \cap t B \neq \varnothing$. Indeed, if there exists an uncountable set $D \subset S$ such that $t \in D$ implies $t B \cap s B \neq \varnothing$, then for a certain element $b \in B$, sb is in uncountably many $t B$, say $s b \in t B$, for $t \in D_{1}$, and $\left|D_{1}\right|>\boldsymbol{\aleph}_{0}$. Since $B$ is countable, there exists $t_{1}, t_{2} \in D_{1}, b_{1} \in B$, such that $t_{1} \neq t_{2}$ and $s b=t_{1} b_{1}=t_{2} b_{1}$. This is impossible, since we have right cancellation on $S$. Thus each equivalence class is at most countable. Let $\left\{A_{\alpha}: \alpha \in \mathfrak{A}\right\}$ be the set of equivalence classes. Thus if $A_{\alpha_{1}} \neq A_{\alpha_{2}}$ and $s_{1} \in A_{\alpha_{1}}, s_{2} \in A_{\alpha_{2}}$ then $s_{1} B \cap s_{2} B=\varnothing$.

Let $S_{\alpha}$ be a fixed equivalence class, say, $S_{\alpha}=\left\{s_{1}, s_{2}, \ldots\right\}$. Set $B\left(s_{1}\right)=s_{1} B, B\left(s_{2}\right)=s_{2} B \backslash s_{1} B, \cdots, B\left(s_{n}\right)=s_{n} B \backslash\left(s_{1} B \cup \cdots \cup s_{n-1} B\right)$, $n>1$. Note that since $B$ is a thin set, $s_{n} B \backslash B\left(s_{n}\right)=\left(s_{n} B \cap s_{1} B\right) \cup \cdots \cup$ $\left(s_{n} B \cap s_{n-1} B\right)$ is finite, hence $B\left(s_{n}\right)^{\wedge}=\left(s_{n} B\right)^{\wedge}$. Also by the construction, if $n \neq m, B\left(s_{n}\right) \cap B\left(s_{m}\right)=\varnothing$. We can do this for each $\alpha \in \mathfrak{A}$. Thus we can construct a family of subsets $B(s), s \in S$, such that $B(s) \subset s B$, $B(s)^{\wedge}=(s B)^{\wedge}$ and if $s \neq t, B(s) \cap B(t)=\varnothing$.

Now let $\omega_{1}, \omega_{2} \in C, \omega_{1} \neq \omega_{2}$. Then we can find a set $B \subset A$ such that $|B|=\boldsymbol{\aleph}_{0}$ and $\omega_{1}, \omega_{2} \in B^{\wedge}$. Let $B(s), s \in S$, be the family of subsets we constructed above with respect to this $B$. For each $s \in S, s^{\sim} \omega_{1}$, 
$s \sim \omega_{2} \in B(s) \wedge$ and $s \sim \omega_{1} \neq s^{\sim} \omega_{2}$. Thus we can find two sets $B_{1}(s)$ and $B_{2}(s)$ with $B_{i}(s) \subset B(s), \quad i=1, \quad 2, \quad B_{1}(s) \cap B_{2}(s)=\varnothing, \quad s^{\sim} \omega_{1} \in B_{1}(s)^{\wedge}$, and $s \sim \omega_{2} \in B_{2}(s)^{\wedge}$. Let $B_{1}=\bigcup\left\{B_{1}(s): s \in S\right\}$ and $B_{2}=\bigcup\left\{B_{2}(s): s \in S\right\}$. Note that $B_{1} \cap B_{2}=\varnothing$. Define a bounded function $f$ on $S$ as follows: $f \equiv 0$ on $B_{1}, f \equiv 1$ on $B_{2}$, and arbitrary elsewhere. Let $\bar{f}$ be the continuous extension of $f$ to $\beta S$. Then $\bar{f}\left(s^{\sim} \omega_{1}\right)=0$ and $\bar{f}\left(s^{\sim} \omega_{2}\right)=1$ for all $s \in S$. Since $\bar{f}$ is continuous, $\bar{f} \equiv 0$ on $o\left(\omega_{1}\right)^{-}$and $f \equiv 1$ on $o\left(\omega_{2}\right)^{-}$. Thus $o\left(\omega_{1}\right)^{-}$ $\cap o\left(\omega_{2}\right)^{-}=\varnothing$.

REMARKs. (1) If $S$ is countable then the proof is much easier and we do not need to assume that $S$ has right cancellation. (2) We have actually proved that if $\omega \in C$ then $o(\omega)^{-}$is homeomorphic to $\beta S$.

6. Proof of Lemma 3. Assume that the conditions in Lemma 3 are true. Set

$$
M(K)=\{\phi \in M l(S): \operatorname{supp} \phi \subset K\} .
$$

The set $M(K)$ is clearly convex, $\omega^{*}$-compact. If we know that $M(K) \neq \varnothing$, then the Krein-Milman Theorem tells us that ex $M(K) \neq \varnothing$. It is also clear that ex $M(K) \subset \operatorname{ex} M l(S)$. Then to complete the proof of Lemma 3 we only need to show that $M(K)$ is nonempty. This can be proved by applying Day's fixed point theorem [2]. But we prefer to give a simple direct proof.

Choose $\psi^{\prime} \in M l^{\prime}(S)$ and $\omega \in K$. We define a new functional $\phi^{\prime}$ on $m(S)$ as follows: for $f \in m(S)$, set $\left(\phi^{\prime}, f\right)=\left(\psi^{\prime}, f^{\sim}\right)$, where $f^{\sim}$ is defined by $f^{\sim}(s)=\bar{f}\left(s^{\sim} \omega\right), s \in S$. Clearly, $\phi^{\prime}$ is a mean on $m(S)$. We claim that $\phi^{\prime}$ is also left invariant. Indeed, for $f \in m(S), s \in S$,

$$
\begin{aligned}
\left(\phi^{\prime}, l_{s} f\right) & =\left(\phi^{\prime},\left(l_{s} f\right)^{\sim}\right)=\left(\psi^{\prime}, l_{s} f^{\sim}\right) \\
& =\left(\psi^{\prime}, f^{\sim}\right)=\left(\phi^{\prime}, f\right) .
\end{aligned}
$$

Finally, we want to show that supp $\phi \subset o(\omega)-\subset K$. Indeed, if $\omega_{1} \notin o(\omega)^{-}$, then there is a closed-open neighborhood $A^{-}, A \subset S$, of $\omega_{1}$ such that $A^{-} \cap o(\omega)^{-}=\varnothing$. Set $f=\chi_{A}$, the characteristic function of the set $A$ in $S$. Note that $\bar{f}=\chi_{A^{-}}$and

$$
\phi\left(A^{-}\right)=\left(\phi^{\prime}, \chi_{A}\right)=\left(\psi^{\prime}, \tilde{\chi_{A}}\right)=0,
$$

since $\tilde{\chi_{A}} \equiv 0$. This clearly implies that $\omega_{1} \notin \operatorname{supp} \phi$.

\section{REFERENCES}

1. M. M. Day, Amenable semigroups, Illinois J. Math. 1 (1957), 509-544.

2. - Fixed-point theorems for compact convex sets, Illinois J. Math. 5 (1961), $585-590$. 
3. L. Gillman and M. Jerison, Rings of continuous functions, Van Nostrand, New York, 1960.

4. E. Granirer, On amenable semigroups with a finite dimensional set of invariant means. I, II, Illinois J. Math. 7 (1963), 32-58.

5. - A theorem on amenable semigroups, Trans. Amer. Math. Soc. 111 (1964), $367-379$.

6. I. S. Luthar, Uniqueness of the invariant mean on an abelian semigroup, Illinois J. Math. 3 (1959), 28-44.

7. C. Wilde and $\mathrm{K}$. Witz, Invariant means and the Stone-Cech compactification, Pacific J. Math. 21 (1967), 577-586.

State University of New York at Buffalo 